\title{
Novel specific microRNA biomarkers in idiopathic inflammatory bowel disease unrelated to disease activity
}

\author{
Jingmei Lin ${ }^{1}$, Noah C Welker ${ }^{2}$, Zijin Zhao ${ }^{1}$, Yong Li ${ }^{1}$, Jianjun Zhang ${ }^{3}$, Sarah A Reuss ${ }^{1}$, \\ Xinjun Zhang ${ }^{4}$, Hwajeong Lee ${ }^{5}$, Yunlong Liu ${ }^{4}$ and Mary P Bronner ${ }^{2}$ \\ ${ }^{1}$ Department of Pathology and Laboratory Medicine, Indianapolis, IN, USA; ${ }^{2}$ Department of Pathology, \\ University of Utah and ARUP Laboratories, Salt Lake City, UT, USA; ${ }^{3}$ Department of Public Health, \\ Indianapolis, IN, USA; ${ }^{4}$ Department of Medical and Molecular Genetics, Center for Computational Biology \\ and Bioinformatics, Indiana University School of Medicine, Indianapolis, IN, USA and ${ }^{5}$ Department of \\ Pathology and Laboratory Medicine, Albany Medical Center, Albany, NY, USA
}

\begin{abstract}
The diagnosis of idiopathic inflammatory bowel disease can be challenging. MicroRNAs (miRNAs) are small, non-coding RNAs that regulate protein synthesis through post-transcriptional suppression. This study is to identify new miRNA markers in inflammatory bowel disease, and to examine whether miRNA biomarkers might assist in the diagnosis of inflammatory bowel disease. Illumina small RNA sequencing was performed on nondysplastic fresh-frozen colonic mucosa samples of the distalmost colectomy tissue from 19 patients with inflammatory bowel disease ( 10 ulcerative colitis and 9 Crohn disease) and 18 patients with diverticular disease serving as controls. To determine differentially expressed miRNAs, the USeq software package identified 44 miRNAs with altered expression (fold change $\geq 2$ and false discovery rate $\leq \mathbf{0 . 1 0}$ ) compared with the controls. Among them, a panel of nine miRNAs was aberrantly expressed in both ulcerative colitis and Crohn disease. Validation assays performed using quantitative reverse transcription PCR (qRT-PCR) on additional frozen tissue from ulcerative colitis, Crohn disease, and control groups confirmed specific differential expression in inflammatory bowel disease for miR-31, miR-206, miR-424, and miR-146a $(P<0.05)$. The expression of these four miRNAs was further evaluated on formalin-fixed, paraffin-embedded tissue of the distalmost colectomy mucosa from cohorts of diverticular disease controls $(n=29)$, ulcerative colitis $(n=36)$, Crohn disease $(n=26)$, and the other diseases mimicking inflammatory bowel disease including infectious colitis $(n=12)$ and chronic ischemic colitis $(n=19)$, again confirming increased expression specific to inflammatory bowel disease $(P<0.05)$. In summary, we demonstrate that miR-31, miR-206, miR-424, and miR-146a are novel specific biomarkers of inflammatory bowel disease. Furthermore, miR-31 is universally expressed in both ulcerative colitis and Crohn disease not only in fresh-frozen but also in formalin-fixed, paraffin-embedded tissues.
\end{abstract}

Modern Pathology (2014) 27, 602-608; doi:10.1038/modpathol.2013.152; published online 20 September 2013

Keywords: colon; Crohn disease; idiopathic inflammatory bowel disease; microRNA; miR-31; next-generation sequencing; ulcerative colitis

Idiopathic inflammatory bowel disease includes ulcerative colitis and Crohn disease, which poses multiple diagnostic challenges based on the overlapping clinical, endoscopic, and histologic findings compared with numerous other forms of enteritis and colitis. The pathogenesis of inflammatory bowel

Correspondence: Dr J Lin, MD, PhD, Department of Pathology and Laboratory Medicine, Indiana University School of Medicine, 350 West 11th Street, Indianapolis, IN 46202, USA.

E-mail: jinglin@iupui.edu

Received 4 January 2013; revised 2 July 2013; accepted 3 July 2013; published online 20 September 2013 disease is not completely understood, but genetic, infectious, physiological, and immunological factors may all have important roles. ${ }^{1,2}$ Unique gene expression profiles have been reported ${ }^{3,4}$ and this study further investigates this opportunity for diagnostic purposes.

MicroRNAs (miRNAs) are encoded within the genomes of a wide variety of eukaryotes, including $>700$ different miRNA genes in the human genome. ${ }^{5,6}$ miRNAs represent a class of regulatory RNAs that suppress gene expression at a post-transcriptional level. miRNAs can concurrently modulate the expression levels of dozens or more distinct 
messenger RNA (mRNA) targets. Alternatively, any given mRNA sequence may be targeted by several different miRNAs. ${ }^{5-7}$ To date, few studies have reported aberrant miRNA expression in inflammatory bowel disease and most have focused on differentiating between ulcerative colitis and Crohn disease. ${ }^{8-13}$

The purpose of this study was to identify novel specific miRNA markers of inflammatory bowel disease in comparison with other common forms of enterocolitis within the differential, including infectious colitis, ischemic colitis, and diverticular disease. Small RNA methodology was used not only on fresh-frozen samples but also on routinely available formalin-fixed, paraffin-embedded tissues.

\section{Materials and methods}

\section{Patients and Human Colonic Tissues}

Fresh-frozen colonic mucosa was sampled from colectomies of the following patient groups: Crohn disease $(n=10)$, ulcerative colitis $(n=20)$, and a 'normal' control group of normal colonic mucosa from diverticular disease patients $(n=18)$. Institutional Review Board approval was strictly maintained. The frozen samples were obtained from the distalmost involved part of the colectomy specimens, snap frozen in liquid nitrogen, and held at $-80{ }^{\circ} \mathrm{C}$. Frozen sections were performed to confirm the non-dysplastic histology and to evaluate the degree of inflammation in each sample.

Colonic mucosa from formalin-fixed, paraffinembedded tissues was dissected from the distalmost

Table 1 Clinical characteristics of patients in the fresh-frozen tissue analysis

\begin{tabular}{lccc}
\hline & Control $^{\mathrm{a}}$ & $C D$ & $U C$ \\
\hline Number of patients & 18 & 9 & 10 \\
Male percentage & $27.8 \%$ & $44.4 \%$ & $60.0 \%$ \\
Age (year, range) & $46(26-74)$ & $42(29-61)$ & $46(31-58)$ \\
Mean duration of & NA & $12.0(1-27)$ & $21.6(9-40)$ \\
IBD (year, range) & & &
\end{tabular}

Abbreviations: IBD, inflammatory bowel disease; CD, Crohn disease; NA, not applicable; UC, ulcerative colitis.

${ }^{\mathrm{a}}$ The control group consists of patients with a diagnosis of diverticular disease. part the colectomies of the following patient groups: Crohn disease $(n=26)$, ulcerative colitis $(n=36)$, infectious colitis (totaling 12 cases including Clostridium difficile colitis $(n=10)$ and cytomegalovirus colitis $(n=2))$, chronic ischemic colitis $(n=19)$, and a 'normal' control group of diverticular disease $(n=29)$. The inflammatory activity of each frozen and formalin-fixed, paraffin-embedded sample was classified into inactive, cryptitis, crypt abscess, numerous crypt abscesses ( $>3$ /high power field), and ulceration. The slides were reviewed by two pathologists (JL and MPB). Clinical characteristics of patients are summarized in Tables 1 and 2 .

\section{Total Small RNA Extraction in Fresh-Frozen Tissue}

The frozen tissues were dissolved in $700 \mu \mathrm{l}$ of Qiazol reagent (Qiagen, Valencia, CA, USA) and total small RNA was extracted using the miRNeasy mini isolation kit (Qiagen), according to the manufacturer's instructions. The RNA samples were stored at $-80^{\circ} \mathrm{C}$.

\section{Genome-Wide Small RNA Sequencing}

Illumina next-generation sequencing of small RNA cDNA libraries was performed at the Genomic Sequencing Core of the Cleveland Clinic on nondysplastic fresh-frozen colonic mucosa samples of the distalmost portion of the colectomy from patients with a diagnosis of ulcerative colitis $(n=10)$, Crohn disease $(n=9)$, and control group $(n=18)$.

\section{Determination of Cutoff Values for miRNA Expression}

Raw sequencing data were aligned to the hg19 build of the human genome with Novoalign using parameters particularly suited for small RNA samples. To determine differentially expressed miRNAs, we used the USeq software package to identify variably expressed miRNAs compared with the controls (fold change, $\geq 2$; false discovery rate, $\leq 0.10$ ).

\section{Total Small RNA Extraction in Formalin-Fixed, Paraffin-Embedded Tissue}

Small RNA was extracted from formalin-fixed, paraffin-embedded tissues using RecoverAll Total

Table 2 Clinical characteristics of patients in the formalin-fixed, paraffin-embedded analysis

\begin{tabular}{|c|c|c|c|c|c|}
\hline & Control $^{\mathrm{a}}$ & $C D$ & $U C$ & Infection $^{\mathrm{b}}$ & Ischemia \\
\hline Number of patients & 29 & 26 & 36 & 12 & 19 \\
\hline Male percentage & $62.1 \%$ & $61.5 \%$ & $72.2 \%$ & $50.0 \%$ & $52.6 \%$ \\
\hline Age (year, range) & $50(31-73)$ & $41(16-68)$ & $46(19-74)$ & $69(52-85)$ & $64(50-79)$ \\
\hline Mean IBD duration (year, range) & NA & $14(0.3-38)$ & $8.5(1-25)$ & NA & NA \\
\hline
\end{tabular}

Abbreviations: CD, Crohn disease; IBD, inflammatory bowel disease; UC, ulcerative colitis; NA, not applicable.

aThe control group consists of patients with a diagnosis of diverticular disease.

${ }^{\mathrm{b}}$ The infection group is composed of 2 patients of cytomegalovirus colitis and 10 patients of $C$. difficile colitis. 
Nucleic Acid Isolation Kits (Ambion by Life Technologies, Grand Island, NY, USA) according to the manufacturer's instructions. Briefly, the formalinfixed, paraffin-embedded tissues were scraped from slides and treated with xylene for deparaffinization. Nucleic acid was then isolated from the tissues by utilizing a protease digestion buffer. After DNase digestion, RNA was purified, recovered, and then stored at $-80^{\circ} \mathrm{C}$.

\section{Quantitative Reverse Transcription PCR}

The miRNA enrichment from the study and control groups was performed using the TaqMan ${ }^{\circledR}$ miRNA Reverse Transcription Kit (Applied Biosystems by Life Technologies, Grand Island, NY, USA) and the TaqMan ${ }^{\circledR}$ Universal PCR Master Mix System (Applied Biosystems). Briefly, $50 \mathrm{ng}$ of small RNA was converted to cDNA. Commercially available miRNA primers were used (Applied Biosystems). The expression of each miRNA in the sampled colonic mucosa was calculated as a value relative to U6B, a ubiquitously expressed small nuclear RNA that has been widely accepted as a stably expressed internal control. The levels of each target miRNA in the study groups were then normalized to the mean value of the control group. Each sample was performed in duplicate.

\section{Statistical Analysis}

Experimental results were expressed as mean values. Quantitative reverse transcription PCR (qRT-PCR) data was analyzed by average fold change in combination with Student's $t$-test. Associations between miRNA expression levels and other parameters (inflammatory activity) were evaluated using Student's $t$-test. A $P$-value of $<0.05$ was considered as significant.

\section{Results}

\section{Identification of Aberrantly Expressed miRNAs in Both Ulcerative Colitis and Crohn Disease Using Genome-Wide Small RNA Sequencing}

Using next-generation sequencing of small RNA cDNA libraries as a discovery platform, 44 miRNAs were identified that were frequently dysregulated in both ulcerative colitis $(n=10)$ and Crohn disease $(n=9)$ in comparison with normal colonic controls (data not shown). As shown in Figure 1, a panel of nine miRNAs (miR-31, miR-206, miR-146a, miR424, miR-663, miR-194b, miR-216b, miR-548e, and miR-559) was aberrantly expressed, either increased or decreased synchronously, in both ulcerative colitis and Crohn disease using stringent comparison criteria relative to the control mucosa (fold change, $\geq 2$; false discovery rate, $\leq 0.10$ ).
Verification of Differential miRNA Expression in Fresh-Frozen Ulcerative Colitis and Crohn Disease Colonic Mucosa Compared With the Normal Control Colonic Mucosa

The expression of nine miRNAs (miR-31, miR-206, miR-146a, miR-424, miR-663, miR-194b, miR-216b, miR-548e, and miR-559) was verified by qRT-PCR in additional frozen colonic mucosal tissue from ulcerative colitis $(n=20)$, Crohn disease $(n=10)$, and the normal colonic control $(n=18)$ groups. The expression of each miRNA was normalized to its internal control, U6B, and then each value in the study groups was standardized to the mean value of the control group. As demonstrated in Figure 2,

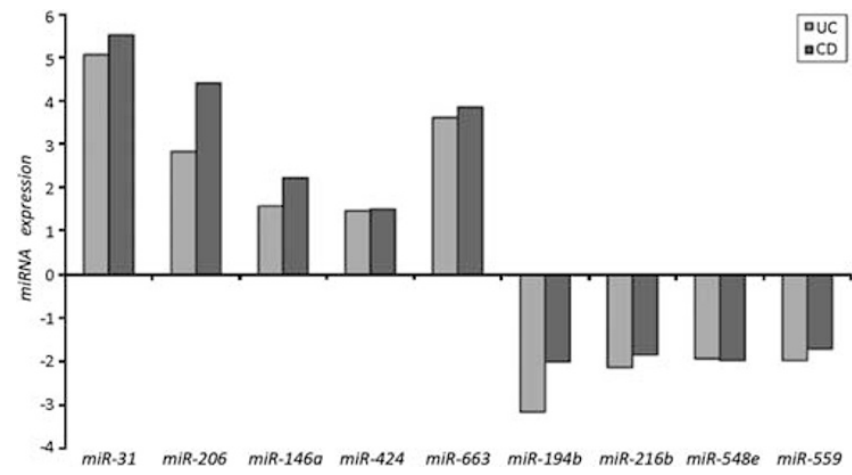

Figure 1 Clustering of nine miRNAs expression profile in ulcerative colitis and Crohn disease using genome-wide small RNA sequencing. Nine miRNAs (miR-31, miR-206, miR-146a, miR-424, miR-663, miR-194b, miR-216b, miR-548e, and miR-559) with aberrant expression, either increased or decreased synchronously, in fresh-frozen colonic mucosa of both ulcerative colitis and Crohn disease compared with the controls (fold change, $\geq 2$; false discovery rate, $\leq 0.10$ ). The control group consists of patients with a diagnosis of diverticular disease.

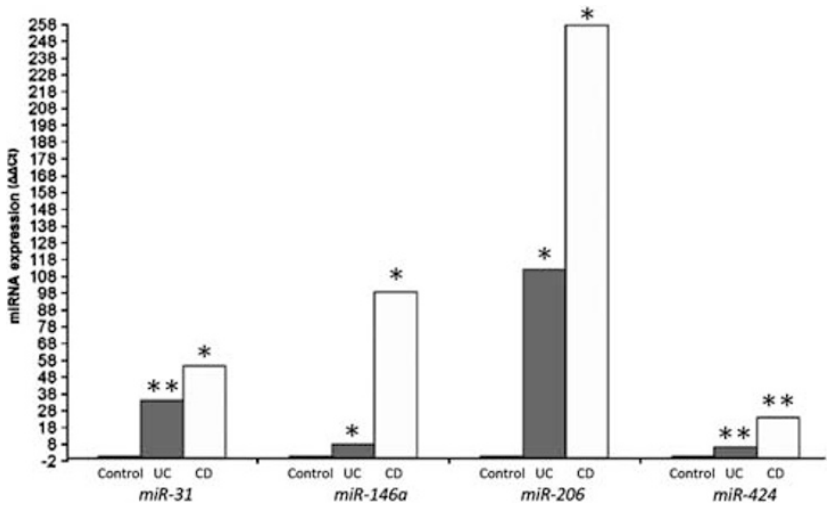

Figure 2 The expression of miR-31, miR-206, miR-424, and miR146a was significantly increased in the fresh-frozen colonic tissue of patients with Crohn disease and ulcerative colitis compared with the normal colonic mucosal controls by qRT-PCR enrichment. The expression of each miRNA was normalized to an internal control, U6B, and then the study groups were standardized to the mean value of the normal colonic mucosal control group. The control group consists of patients with a diagnosis of diverticular disease. ${ }^{*} P<0.05$ compared with the control group; ${ }^{*} P<0.01$ compared with the control group. 
analysis of variance showed a significant difference in the expression of miR-31, miR-146a, miR-206, and miR-424 in both Crohn disease and ulcerative colitis groups compared with the control group $(P<0.05$ and $P<0.01$, respectively). No differences were observed in the rest of the five miRNAs (miR663, miR-194b, miR-216b, miR-548e, and miR-559) in Crohn disease and ulcerative colitis compared with the control groups ( $P>0.05$; data not shown).

\section{Formalin-Fixed, Paraffin-Embedded Tissue Validation}

The expression of four differentially expressed miRNAs (miR-31, miR-146a, miR-206, and miR424) was further evaluated in the formalin-fixed, paraffin-embedded colonic mucosal tissue of additional inflammatory bowel disease patients (ulcerative colitis $(n=36)$ and Crohn disease $(n=26))$ and control groups $(n=29)$. The expression of each miRNA was normalized to its internal control, $\mathrm{U6B}$, and then each value in the study groups was standardized to the mean value of the control group. As shown in Figure 3a, there was a significant
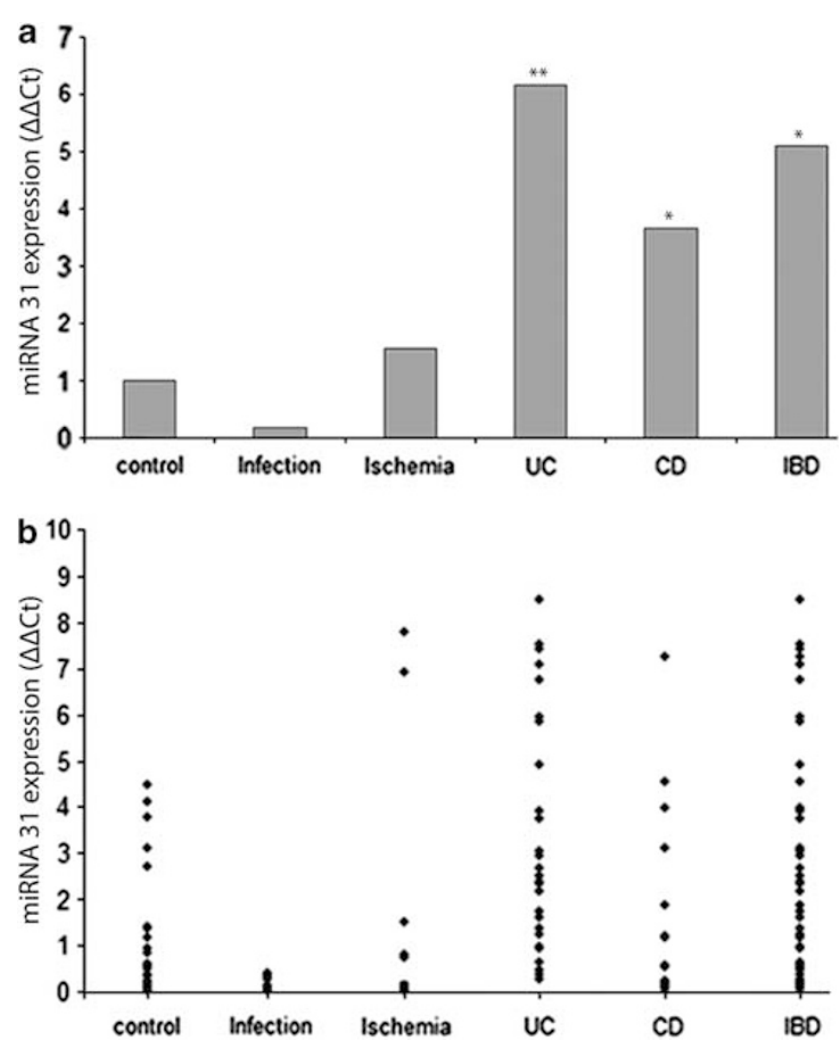

Figure 3 (a) The mean and (b) absolute expression levels of miR-31 from formalin-fixed, paraffin-embedded distalmost colonic mucosa of patients with ulcerative colitis $(n=36)$ or Crohn disease $(n=26)$ compared with normal colonic mucosal controls $(n=29)$ by qRT-PCR enrichment. miR-31 expression in each sample was normalized to an internal control, U6B, and then to the mean value of the normal colonic mucosal control group. The control group consists of patients with a diagnosis of diverticular disease. ${ }^{*} P<0.05$ compared with the control group; ${ }^{* *} P<0.01$ compared with the control group. difference in the expression of miR-31 in Crohn disease and ulcerative colitis groups, either individually or as a group of inflammatory bowel disease, compared with the control group $(P<0.05$ and $P<0.01$, respectively). However, the other three miRNAs (miR-206, miR-424, and miR-146a) showed no differential expression compared with the control group in formalin-fixed, paraffin-embedded tissue $(P>0.05$; data not shown).

Differential Expression of miR-31 in Formalin-Fixed, Paraffin-Embedded Colonic Tissue in Both Ulcerative Colitis and Crohn Disease Patients Compared With The Controls and The Other Diseases Mimicking Inflammatory Bowel Disease

As shown in Figures 3a and b, the expression of miR-31 was also evaluated in the formalin-fixed, paraffin-embedded colonic mucosal tissue from diseases mimicking inflammatory bowel disease, including infectious colitis $(n=12$, including Clostridium difficile $(n=10)$ and cytomegalovirus colitis $(n=2))$ and chronic ischemic colitis $(n=19)$. As shown in Table 3 , analysis of variance showed significant differences in the expression of miR-31 in Crohn disease and ulcerative colitis groups, either individually or as a group of inflammatory bowel disease, compared with the infectious or ischemic groups, respectively $(P<0.05$ and $P<0.01)$, with an exception between Crohn disease and ischemia groups.

\section{Inflammatory Activity Grade in Inflammatory Bowel Disease and Controls}

The degree of active inflammation in each frozen and formalin-fixed, paraffin-embedded sample was evaluated. As shown in Tables 4 and 5, most controls (either frozen tissues or formalin-fixed paraffinembedded) showed no evidence of inflammation; however, most of the specimens of the study groups, including Crohn disease and ulcerative colitis (either frozen tissues or formalin-fixed, paraffinembedded) demonstrated variable degrees of inflammation, ranging from cryptitis to crypt abscess to ulceration. The infectious disease and ischemia

Table 3 The $P$-values in the analysis of formalin-fixed, paraffinembedded tissues

\begin{tabular}{lccc}
\hline & $\begin{array}{c}\text { Control }^{\mathrm{a}} \\
(\mathrm{n}=29)\end{array}$ & $\begin{array}{c}\text { Infection } \\
(\mathrm{n}=12)\end{array}$ & $\begin{array}{c}\text { Ischemia } \\
(\mathrm{n}=19)\end{array}$ \\
\hline $\mathrm{UC}(n=36)$ & 0.00034 & 0.00006 & 0.00217 \\
$\mathrm{CD}(n=26)$ & 0.04644 & 0.01482 & 0.10703 \\
$\mathrm{IBD}(n=62)$ & 0.00010 & 0.00001 & 0.00261 \\
\hline
\end{tabular}

Abbreviations: CD, Crohn disease; IBD, inflammatory bowel disease; UC, ulcerative colitis.

${ }^{\mathrm{a}}$ The control group consists of patients with a diagnosis of diverticular disease. 
Table 4 Inflammatory activity in frozen samples studied by small RNA sequencing

\begin{tabular}{lccccc}
\hline & $\begin{array}{c}\text { Inactive } \\
(\%)\end{array}$ & $\begin{array}{c}\text { Cryptitis } \\
(\%)\end{array}$ & $\begin{array}{c}\text { Crypt } \\
\text { abscess } \\
(\%)\end{array}$ & $\begin{array}{c}\text { Numerous } \\
\text { crypt } \\
\text { abscesses }\end{array}$ & Ulcer \\
\hline $\begin{array}{l}\text { Controls }^{\mathrm{a}} \\
(n=18)\end{array}$ & 77.8 & 16.7 & 5.6 & 0 & 0 \\
$\mathrm{CD}(n=9)$ & 11.1 & 77.8 & 11.1 & 0 & 0 \\
$\mathrm{UC}(n=10)$ & 10 & 60 & 20 & 0 & $10 \%$ \\
\hline
\end{tabular}

Abbreviations: CD, Crohn disease; UC, ulcerative colitis.

${ }^{\mathrm{a}}$ The control group consists of patients with a diagnosis of diverticular disease.

Table 5 Inflammatory activity in formalin-fixed, paraffin-embedded tissues

\begin{tabular}{lccccc}
\hline & $\begin{array}{c}\text { Crypt } \\
\text { Inactive } \\
(\%)\end{array}$ & $\begin{array}{c}\text { Cryptitis } \\
(\%)\end{array}$ & $\begin{array}{c}\text { Numerous } \\
\text { abscess } \\
(\%)\end{array}$ & $\begin{array}{c}\text { crypt } \\
\text { abscess }(\%)\end{array}$ & $\begin{array}{c}\text { Ulcer } \\
(\%)\end{array}$ \\
\hline $\begin{array}{l}\text { Controls } \\
(n=29)\end{array}$ & 100 & 0 & 0 & 0 & 0 \\
CD $(n=26)$ & 84.6 & 3.8 & 0 & 0 & 11.5 \\
UC $(n=36)$ & 8.3 & 11.1 & 17.1 & 5.7 & 58.3 \\
$\begin{array}{l}\text { Infection } \\
(n=12)\end{array}$ & 8.3 & 33.3 & 16.7 & 8.3 & 33.3 \\
$\begin{array}{l}\text { Ischemia } \\
(n=19)\end{array}$ & 78.9 & 15.8 & 5.3 & 0 & 0 \\
\hline
\end{tabular}

Abbreviations: CD, Crohn disease; UC, ulcerative colitis.

${ }^{a}$ The control group consists of patients with a diagnosis of diverticular disease.

groups encompassed some activity with the levels between that of ulcerative colitis and Crohn disease; however, the miR-31 expression level of these two groups is as low as or even lower than the controls, which speaks against the assumption that the expression of miR-31 is correlated with inflammatory activity grade. Thus, the expression levels of miRNAs were not associated with the degree of inflammation in the current study $(P>0.05$; data not shown).

\section{Discussion}

While ulcerative colitis and Crohn disease differ clinically, radiographically, serologically, endoscopically, and histologically, they frequently overlap clinically, and have also been shown to share common gene expression patterns. ${ }^{13-18}$ In this study, we identified miR-31, miR-146a, miR-206, and miR-424 as novel miRNA biomarkers that are commonly and specifically expressed in the freshfrozen tissue of colonic mucosa from inflammatory bowel disease patients compared with colonic mucosa control patients. The dysregulated expression of four miRNAs in both ulcerative colitis and Crohn disease suggests that these miRNAs might be involved in the regulation of the common

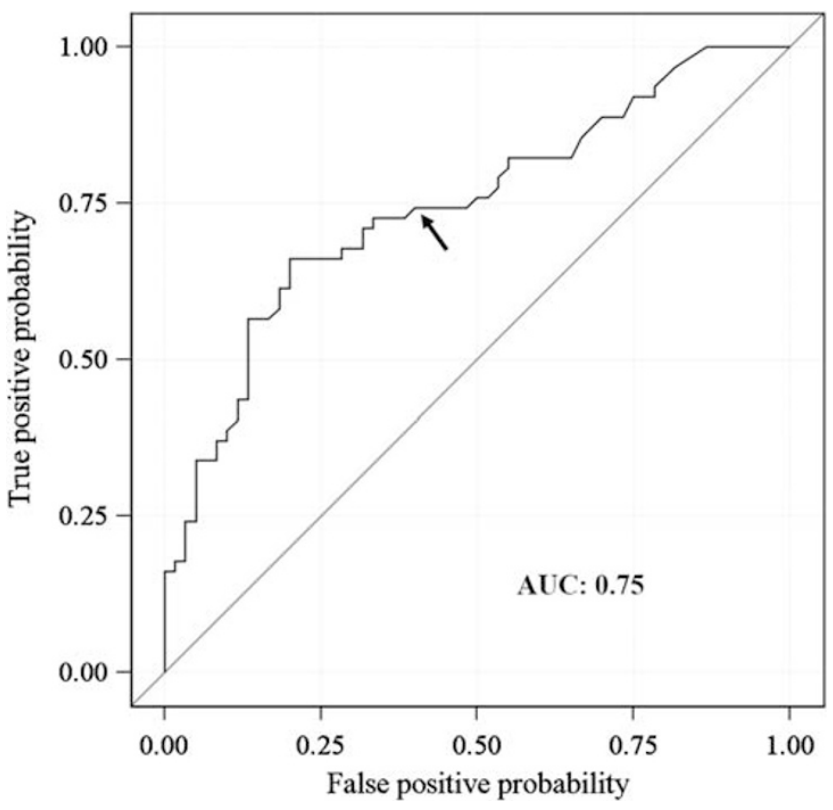

Figure 4 ROC curve of miR-31 in diagnosing inflammatory bowel disease from formalin-fixed, paraffin-embedded distalmost colonic mucosa by qRT-PCR enrichment. Arrow: cutoff point on the ROC curve for 0.5 of relative value miR-31 where the sensitivity is $71.0 \%$ and specificity is $67.2 \%$.

pathophysiological pathway leading to inflammatory bowel disease. However, the differential expression of three miRNAs (miR146a, miR-206, and miR-424) was not confirmed in formalin-fixed, paraffin-embedded tissue. A possible explanation is that the signals are lost or altered during processing, or these three miRNAs are not true specific markers of inflammatory bowel disease.

The most important finding in this study is that miR-31 is constantly upregulated in both freshfrozen and formalin-fixed, paraffin-embedded tissues of colonic mucosa in both ulcerative colitis and Crohn disease patients, with an overall sensitivity of $71.0 \%$ and a specificity of $67.2 \%$ in formalin-fixed, paraffin-embedded tissue. As shown in Figure 4, ROC curve, a measurement of accuracy, shows an AUC of 0.75 , which suggests a moderate predictive accuracy of miR-31 in diagnosing inflammatory bowel disease. MiR-31, preserved in vertebrates and Drosophila, is a pleiotropically acting miRNA that is expressed in a variety of tissues and cell types. ${ }^{19,20}$ MiR-31 dysregulation has been suggested to be associated with malignancy and metastatic disease ${ }^{21,22}$ however, its role in the non-dysplastic aspect of inflammatory bowel disease has not been generally recognized. ${ }^{21,22}$ Aberrant expression of miRNA-31 has been briefly mentioned in inflammatory bowel disease patients in a prior study of inflammatory bowel disease-associated colon cancer, but it has not been recognized as a specific marker in inflammatory bowel disease. ${ }^{13}$ In this study, we emphasize the role of miR-31 as a universal biomarker of idiopathic inflammatory bowel 
disease regardless the grade of inflammatory activity. Our study also shows the utility of this marker in routinely available formalin-fixed, paraffin-embedded tissue. We provide evidence that miR-31 shows promise as a diagnostic biomarker of inflammatory bowel disease. It might be used to differentiate inflammatory bowel disease from its mimics, including infectious colitis, chronic ischemic colitis, diverticular disease-associated colitis, drug/medicationinduced colitis, or colitis of unknown cause. Often the evaluation of the pathology in these colectomies can be hampered or impossible due to the extensive ulceration. However, the diagnosis of inflammatory bowel disease is of critical clinical importance in terms of the proper clinical management, since the inflammatory bowel disease patients have an increased risk of developing colorectal cancer, ${ }^{23}$ while the non-inflammatory bowel disease patients are largely free of the risk and are spared from difficult and costly cancer surveillance.

This study brings several advances, as (1) the first report assessing miR-31 expression in inflammatory bowel disease in formalin-fixed, paraffin-embedded tissue; (2) the first report to identify aberrant expression of miR-31 in inflammatory bowel disease independent of inflammatory activity; and (3) an addition to small but growing knowledge of miRNA analysis by next-generation sequencing in colonic mucosa of inflammatory bowel disease patients. However, there are several potential limitations to the use of miRNA in the diagnosis of inflammatory bowel disease. Additional studies to address the spatial distribution of miRNA throughout the colon and optimal biopsy sampling are needed. The level of miR-31 expression has been reported to be steady and parallel throughout the colon from cecum to rectum in frozen tissues of normal volunteers without history of inflammatory bowel disease in a study of inflammatory bowel disease-associated colon cancer; ${ }^{21}$ in other words, the current knowledge from this study indicates that the variable expression levels of miR-31 in inflammatory bowel disease patients likely reflect the disease status itself rather than location bias, although further verification is needed in subsequent publications.

Second, inflammatory activity is another consideration given the well-documented viable inflammatory condition of the disease. All miRNA candidates in this study were identified from nextgeneration sequence analysis in which the colonic mucosa of both ulcerative colitis and Crohn disease patients encompassed variable inflammatory activity. In the study, the expression of miR-31 has been verified in both fresh-frozen and formalin-fixed, paraffin-embedded mucosal tissue, regardless the inflammatory status of the samples. Thus, we suggest miR-31 as a universally expressed biomarker of inflammatory bowel disease, regardless the location or the degree of inflammatory activity.

Finally, the treatment effect on miRNA expression profile on colonic mucosa is largely unknown. Most of these inflammatory bowel disease patients in the study underwent extensive medical regimens and were unresponsive to treatment before surgical intervention. Of these patients, the clinical course and regimens were different. Due to the size limitation, we do not have enough samples to perform statistical analysis in the current study, although we agree that it is an important issue, or at least we cannot exclude such possibility. The study of altered miRNA expression profile in inflammatory bowel disease has emerged recently; there is so much we do not know. The more attention and studies in this field will be helpful to elucidate the underlying mechanisms of miRNA in inflammatory bowel disease and provide insight in treatment.

In summary, miRNAs show promise as diagnostic markers in inflammatory bowel disease. This study provides evidence for the moderate sensitivity and specificity of miR-31 in inflammatory bowel disease, including both ulcerative colitis and Crohn disease, relative to the common non-inflammatory bowel disease mimics of diverticular disease, chronic infectious colitis, and ischemic colitis.

\section{Acknowledgements}

We would like to thank Bonnie Shadrach and Jennifer Lewis for their help in procuring the frozen tissue and Kerra Wagener, Tracey Bender, and Amy Thomasson for their assistance in the preparation of this manuscript.

\section{Disclosure/conflict of interest}

The authors declare no conflict of interest.

\section{References}

1 Xavier RJ, Podolsky DK. Unravelling the pathogenesis of inflammatory bowel disease. Nature 2007;448: 427-434.

2 Kugathasan S, Amre D. Inflammatory bowel diseaseenvironmental modification and genetic determinants. Pediatr Clin North Am 2006;53:727-749.

3 Lawrance IC, Fiocchi C, Chakravarti S. Ulcerative colitis and Crohn's disease: distinctive gene expression profiles and novel susceptibility candidate genes. Hum Mol Genet 2001;10:445-456.

4 Abraham C, Cho JH. Inflammatory bowel disease. N Engl J Med 2009;361:2066-2078.

5 Ambros V. The functions of animal microRNAs. Nature 2004;431:350-355.

6 Bartel DP. MicroRNAs: target recognition and regulatory functions. Cell 2009;136:215-233.

7 Saini HK, Enright AJ, Griffiths-Jones S. Annotation of mammalian primary microRNAs. BMC Genomics 2008;9:564.

8 Lin J, Cao Q, Zhang J, et al. MicroRNA expression patterns in indeterminate inflammatory bowel disease. Mod Pathol 2013;26:148-154.

9 Takagi T, Naito Y, Mizushima K, et al. Increased intestinal expression of heme oxygenase-1 and its 
localization in patients with ulcerative colitis. J Gastroenterol Hepatol 2008;23(suppl 2):S229-S233.

$10 \mathrm{Wu} F$, Zhang S, Dassopoulos T, et al. Identification of microRNAs associated with ileal and colonic Crohn's disease. Inflamm Bowel Dis 2010;16:1729-1738.

11 Wu F, Zikusoka M, Trindade A, et al. MicroRNAs are differentially expressed in ulcerative colitis and alter expression of macrophage inflammatory peptide-2 alpha. Gastroenterology 2008;135:1624-1635; e24.

12 Duttagupta R, DiRienzo S, Jiang R, et al. Genome-wide maps of circulating miRNA biomarkers for ulcerative colitis. PLoS ONE 2012;7:e31241.

13 Fasseu M, Treton X, Guichard C, et al. Identification of restricted subsets of mature microRNA abnormally expressed in inactive colonic mucosa of patients with inflammatory bowel disease. PLoS ONE 2010;5: pii: e13160.

14 Fujino S, Andoh A, Bamba S, et al. Increased expression of interleukin 17 in inflammatory bowel disease. Gut 2003;52:65-70.

15 Zhang T, Song B, Zhu W, et al. An ileal Crohn's disease gene signature based on whole human genome expression profiles of disease unaffected ileal mucosal biopsies. PLoS ONE 2012;7:e37139.

16 Mesko B, Poliska S, Szegedi A, et al. Peripheral blood gene expression patterns discriminate among chronic inflammatory diseases and healthy controls and identify novel targets. BMC Med Genomics 2010;3:15.
17 Berberian LS, Valles-Ayoub Y, Gordon LK, et al. Expression of a novel autoantibody defined by the VH3-15 gene in inflammatory bowel disease and Campylobacter jejuni enterocolitis. J Immunol 1994; 153:3756-3763.

18 Clark PM, Dawany N, Dampier W, et al. Bioinformatics analysis reveals transcriptome and microRNA signatures and drug repositioning targets for IBD and other autoimmune diseases. Inflamm Bowel Dis 2012;18: 2315-2333.

19 Landgraf P, Rusu M, Sheridan R, et al. A mammalian microRNA expression atlas based on small RNA library sequencing. Cell 2007;129:1401-1414.

20 Grimson A, Farh KK, Johnston WK, et al. MicroRNA targeting specificity in mammals: determinants beyond seed pairing. Mol Cell 2007;27:91-105.

21 Olaru AV, Selaru FM, Mori Y, et al. Dynamic changes in the expression of MicroRNA-31 during inflammatory bowel disease-associated neoplastic transformation. Inflamm Bowel Dis 2011;17:221-231.

22 Valastyan S, Weinberg RA. miR-31: a crucial overseer of tumor metastasis and other emerging roles. Cell Cycle 2010;9:2124-2129.

23 Jiang W, Shadrach B, Carver P, et al. Histomorphologic and molecular features of pouch and peripouch adenocarcinoma: a comparison with ulcerative colitisassociated adenocarcinoma. Am J Surg Pathol 2012; $36: 1385-1394$. 